Original Research Paper

\title{
Management of Source-Sink Balance for Maintaining Seed Vigor and Storability of Maize
}

\author{
${ }^{1}$ Sirinthorn Kaewchuai, ${ }^{1 *}$ Pitipong Thobunluepop, ${ }^{1}$ Sutkhet Nakasathien, \\ ${ }^{1}$ Sukumarn Lertmongkol, ${ }^{1}$ Damrongvudhi Onwimol, ${ }^{3}$ Wilasinee Chitbanchong and ${ }^{2}$ Michael Bredemeier \\ ${ }^{1}$ Department of Agronomy, Faculty of Agriculture, Kasetsart University, Bangkok 10900, Thailand \\ ${ }^{2}$ Forest Ecosystems Research Group, Center for Biodiversity and Sustainable Land Use (CBL) Universität Göttingen, \\ Buesgenweg 1, D-37077 Göttingen, FRG, Germany \\ ${ }^{3}$ Botany and Herbarium Research Group, Plant Varieties Protection Office, Department of Agriculture, \\ Ministry of Agriculture and Cooperatives, Royal Thai Government, Bangkok, 10900, Thailand
}

Article history

Received: 28-09-2020

Revised: 23-11-2021

Accepted: 08-12-2020

Corresponding Author: Pitipong Thobunluepop Department of Agronomy, Faculty of Agriculture, Kasetsart University, Bangkok 10900, Thailand

Email: fagrppt@ku.ac.th pitipongtho@yahoo.com

\begin{abstract}
Defoliation was the major factor which directly affected to maize seed yield and quality basis on source-sink relationship. Thus, the objectives were to study the effect of source-sink balance management on corn seed vigor and storability and to determine the effect of defoliation treatments on maize yield, yield components and produced seed germination traits. The experiment was arranged basis on Split-Plot in Randomized Complete Block Design with four replications. Main plot was the pattern of leave cutting with 5 levels: $D_{1}=$ control, (without defoliating), $\mathrm{D}_{2}=$ complete defoliation, $\mathrm{D}_{3}=$ defoliating only under the ear, $\mathrm{D}_{4}=$ remain 2 top leaves, $\mathrm{D}_{5}=$ remain ear leaf. Sup-plot was leaf cutting dates which were $C_{1}=7$ days after silking, $C_{2}=10$ days after silking, $\mathrm{C}_{3}=13$ days after silking and $\mathrm{C}_{4}=16$ days after silking. Complete defoliation severely reduced ear weight, row number per ear, seed number per ear and 100 - seed weight $(\mathrm{P}<5 \%)$. Defoliation treatments had much more significantly affected on produced seed germination and seed vigor traits. Leaf defoliation intensity and leaf position affected total dry matter. Conclusion, the leaf defoliation only below ears was useful for source-sink balance management, because it promoted the seed qualities and vigor following to principle of parasitic sink elimination.
\end{abstract}

Keywords: Maize, Source, Sink, Vigor and Storability

\section{Introduction}

Source capacity was determined by photosynthetic activity which related to availability of carbohydrate reserves (Uhart and Andrade, 1991). During effective grain filling period, the interaction between source capacity and sink strength (i.e., the source/sink ratio) would result in variation of final grain weight (Borrás and Otegui, 2001). Middle leaves of the stem had greater important role than the other leaves because of greater surface for light absorbing in the photosynthesis. Completely defoliation was led to minimum seeds yield because of decrease in seed weight and filled grain percent (Gifford et al., 1984). Distance of leaves and ear which participant in photosynthetic efficiency were important in a slight defoliation. Top leaves of the ear transferred about 23 to 91 percent of photosynthates to the cob and the greatest number of transferred materials belonged to the nearest leaf on top of the ear (Collantes et al., 1997). Defoliation treatments imposed when the numbers of grains had been established to reduce the source/sink ratio then results in a sharp decreasing of soluble carbohydrates in stems (Uhart and Andrade, 1995).

According to (Borrás et al., 2004), the imbalance of source/sink ratio during post-flowering could dramatically reduce final kernel weight. Restricting the source capacity during the effective grain-filling period effected Kernel Water Content (KWC) and the differently dynamics of dry matter deposition. Shortage of assimilate availability per grain (post-flowering source/sink ratio) in case that promoted by low irradiance values or defoliation might be reflected on both kernel weight and kernel composition (Borrás et al., 2002). The analysis source/sink ratio during post-flowering stage which effect on kernel weight determination would improve the understanding of the magnitude and source limitations during grain filling of maize (Borrás et al., 2009). The short period of maize leaf defoliation up to $50 \%$ did not had an adverse effect on 
maize grain and yield components (van den Boogaard et al., 2001). Remove of leaves in the pollination phase decreased dry matter and grain yield of maize significantly (Borrás et al., 2004).

Seed development was the period between fertilization, maximum fresh weight accumulation and seed maturation; it began at the end of seed development and continues till harvested (Mehta et al., 1993). Optimum harvesting period at seed matured helps to obtain better seed quality and harvesting stage influenced the quality of seed which related to germination, vigor, viability and storability. Storability of seeds was a major genetically character and is influenced by pre-storage history of seed, seed maturation and environmental actors during pre/post-harvest stages (Shaheb et al., 2015). Early harvested seeds would be low seed quality caused by immature and poorly developed and poor storage compared to seed harvest at physiological maturity (Khatun et al., 2009). Storability of seeds was influenced by pre-storage history of the seeds, seed maturation and environmental factors during pre-harvest and post-harvest (Tuite and Foster, 1979).

\section{Materials and Methods}

The experiment was supported by Syngenta seed (Thailand) Co. Ltd.in research place and plant material, two experiments were conducted at the experimental field where located at U-Thong district, Suphan Buri province, Thailand. During the 2018 and 2019 growing seasons.

\section{Plant Sampling Management and Experimental Design}

In 2018, $F_{1}$ maize hybrids were planted 10 December the experiment was arranged into Split-plot in Randomized Complete Block Design with four replications.

Main-plot: The pattern of leave cutting with 5 levels were $\mathrm{D}_{1}=$ control, (without defoliation), $\mathrm{D}_{2}=$ complete defoliation, $\mathrm{D}_{3}=$ defoliating leaves below the ear, $\mathrm{D}_{4}=$ remain 2 top leaves, $\mathrm{D}_{5}=$ remain ear leaf.

Sub-plot: Leaf cutting date with 4 levels were $C_{1}=7$ days after silking, $C_{2}=10$ days after silking, $C_{3}=13$ days after silking and $\mathrm{C}_{4}=16$ days after silking.

In 2019, hybrids were planted 9 May in plots, again into Split-plot in Randomized Complete Block Design with four replications.

Plant materials and seed samples were $F_{1}$ hybrid maize seeds collected from parental hybrids lines for production. Plants were grown by $20 \times 40 \mathrm{~cm}$. of crop spacing. Compound fertilizer (15-15-15) at $60 \mathrm{~kg} \mathrm{~h}^{-1}, 40 \mathrm{~kg} \mathrm{~h}^{-1}$ after 3-5 days of germination, $20 \mathrm{~kg} \mathrm{~h}^{-1}$ after 40 days of emergence was applied for topping fertilizer single fertilizer (46-0-0) was applied as basal fertilizer amount 50 to $30 \mathrm{~kg} \mathrm{~h}^{-1}$ divided at 20 days after emergence and 15 $\mathrm{kg}$ after $\mathrm{h}^{-1} 40$ days after emergence. Droplet irrigation was applied once a week. Weeds were controlled herbicides by spraying (2.4-D, glufosinate1.0 $\mathrm{L} \mathrm{h}^{-1}+$ fluroxypyr $0.3 \mathrm{~L} \mathrm{~h}^{-1}$ ).

Plants were harvested when at 110 day after emergence. After each harvest, samples containing 10 ears were placed in paper bags and then taken to a hot air oven for drying at $40^{\circ} \mathrm{C}$. The drying was performed until the seeds reached approximately $12 \%$ of moisture content. Seeds were stored in plastic sealed bag in $25^{\circ} \mathrm{C}$. Then, seeds were sampling for seed qualities and vigor was tested at 6th month.

For the sampling, five plants were used as represent from each sampling block then separated each plant part and dried. Data collection was consisting of seed yield (gram), row number per cob, seed number per row and ear weight and 100 -seed weight with remarkably that seed samples were bulk in each sampling block before the measurement.

\section{Seed Parameters}

Seeds of maternal plants were stored for $6^{\text {th }}$ month then used for the seed parameters measurement, the effect of maternal environment was studied by testing seed germination traits. Seed qualities were tested following.

\section{Determination of Germination Percentage}

Germinations were carried out according to (ISTA, 2020). For each treatment, 100 seeds were germinated by using between paper techniques with four replications. The rolled papers were cultivated at room temperature $\left(25 \pm 2^{\circ} \mathrm{C}\right)$. After the first count and final count 4 and 7 days after germination, normal, abnormal and diseased seeds were counted. Seed germination was calculated by the following formula:

$$
\text { Seed } \text { germination }(\%)=\frac{\text { No. of seeds } \text { germinated }}{\text { Total } \text { seeds }} \times 100
$$

\section{Measurement of Root and Shoot Length}

Final count, five seedlings were randomly selected as a represent for study, taking from each replicate of each treatment. The seedlings were cut into root and shoot parts and their lengths were measured as centimeter $(\mathrm{cm})$.

\section{Determination of Seed Vigor}

Seedling vigor parameters were testing followed up protocols which were determined by ISTA: The Accelerated Aging test (AA) (ISTA, 2020), speed of germination (ISTA, 2020) and seedling growth rate (ISTA, 2020). These tests would predict storage and field planting potential. High humidity and high temperature stress were imposed on the seed, which was incubated for a period under these conditions, then transferred to a growth chamber to assess germination potential. Seed lots that withstand these conditions, while maintaining a 
germination rate of $90 \%$ or above, are considered high vigor (ISTA, 2020).

\section{Speed of Germination}

This parameter was calculated by the following formula given by ISTA, (2020):

$$
\text { Speed of germination }=n 1 / d 1+n 2 / d 2+n 3 / d 3
$$

Where:

$n=$ Number of germinated seeds

$d=$ Number of days

\section{Seedling Growth Rate Test}

This test was closely related to the standard germination test and is useful to figure out field planting potential under optimal or near ideal conditions. Seeds were planted under optimal condition and promoted to grow for an extended period, usually several days past the typical germination period. The seedlings are evaluated by their growth characteristics, such as stem length, leaf development or root branching (ISTA, 2020).

The data were submitted to the Analysis Of Variance (ANOVA). Using a Split plot in Randomized Complete Block Design with 4 replications, mean comparisons were accomplished using a Least Significant Difference (LSD) test at the 5\% level. Simple correlation analysis between the results obtained from each test method was conducted.

\section{Results and Discussion}

\section{Ear Weight}

Completely defoliation severely reduced ear weight in both years 2018 and 2019 (Table 1 and 4). Defoliating leaves under of the ear had greater ear weight than removing top leaves of the ear. Maybe it was due to that ear leaf acted as a parasitic sink for ear growth at grain filling period because it was in middle part of maize stem then easily shade on it. Reduction of leaf area reduced resources for grain filling (Koptur et al., 1996). According to leaf cutting date, leaves cut at defoliation in 13 days after silking $\left(\mathrm{C}_{3}\right)$ showed the highest ear weight (Table 4). A decreasing of source in the post-flowering source/sink ratio could reduce final kernel weight dramatically (Borrás et al., 2004). Ear weight was decreased significantly by early defoliation treatment (13 days after silking in both years had greater ear weight than 7 and 10 days after silking). Ear weight had shown to vary with environmental conditions that directly affect to plant growth and assimilate supply per kernel during the period when plants are setting their kernels (i.e., flowering) (Gambín et al., 2006). Differences in ear weight among hybrids and years were mostly affected by differences in the rate of kernel growth, as there were no differences in the duration of grain filling (Table 1 and 4).

\section{Row Number Per Ear and Seed Number Per Row}

Completely defoliation severely decreased row number per ear and seed number per row in both years 2018 and 2019 (Table 1 and 4). Reduction of supply assimilation by defoliations had significantly reduced row number per ear and seed number per row. Heidari (2012) reported, the row number per ear was harmful by complete defoliation. Minor effect of defoliation on seed number per row and row number per ear was due to that stem reserves can compensate insufficient photosynthesis from leaves. Defoliating top leaves of the ear produced lower seed number per row than defoliating leaves below ear. Upper leaves could be available to receive greater light than lower leaves, so defoliation of upper leaves had more adverse effect on seed number per row than lower leaves. Interaction between defoliation and leaf cutting date did significantly alter seed number per row concentration in 2018 year (Table 2). Interaction of D3 and C3 showed the highest row number per ear which was statistically significant (Table 5).

\section{0-Seed Weight}

The result of both years 2018 and 2019 showed, Removing all leaves severely reduced seed yield (Table 1 and 4). Defoliating leaves below the ear had greater amount of seed yield than defoliating leaves at the top of ear $\left(D_{4}\right.$, $\mathrm{D}_{5}$ ). It was probably due to that ear leaf in central part of maize stem and upper leaves can shade on it, so it becomes consumer and competes with ear for photosynthates. Lower seed yield of complete defoliation treatment was due to lower seed number per row and lower row number per ear. Defoliation treatments had significantly affected on 100seed weight (Table 1 and 4) as same as the observed that defoliation decreased seed weight. It seems that seed weight is more dependent on genetic factors than environmental factors (Heidari, 2012).

\section{Seed Germination Percentage}

$\mathrm{D}_{1}$ and $\mathrm{D}_{3}$ had higher seed germination percentage than $D_{2}, D_{4}$ and $D_{5}$ in both years 2018 and 2019 (Table 3 and 6). It might be due to that defoliation as an environmental stress can reduce seed germination percentage as described by (Heidari, 2012). Defoliation of leaves top ear $\left(\mathrm{D}_{4}\right.$ and $\left.\mathrm{D}_{5}\right)$ had no effected as great as a defoliation of leaves under ear $\left(D_{3}\right)$. The reason was bottom leaves was currently senescent and available to receive low light so removal of them does have great effect on plant growth. According to leaf cutting date, leaves cut at defoliation in 13 days after silking $\left(\mathrm{C}_{3}\right)$ showed the highest seed germination percentage (Table 6). 
Table 1: Effect of defoliation patterns and leaf cutting date on total maize yield and yield components during 2018 (year 1)

\begin{tabular}{|c|c|c|c|c|}
\hline Treatment $^{1 /}$ & Ear weight, g/plant b & Row number per ear & Seed number per row & 100 - Seed weight \\
\hline \multicolumn{5}{|l|}{ Defoliation } \\
\hline $\mathrm{D}_{1}$ & $48.50 \mathrm{a}$ & $12.58 \mathrm{a}$ & $14.47 \mathrm{a}$ & $26.84 \mathrm{a}$ \\
\hline $\mathrm{D}_{2}$ & $27.75 b$ & $9.96 \mathrm{~b}$ & $9.77 \mathrm{~b}$ & $10.99 \mathrm{c}$ \\
\hline $\mathrm{D}_{3}$ & $48.00 \mathrm{a}$ & $12.21 \mathrm{a}$ & $14.34 \mathrm{a}$ & $28.84 \mathrm{a}$ \\
\hline $\mathrm{D}_{4}$ & $40.75 \mathrm{a}$ & $12.03 \mathrm{a}$ & $13.96 \mathrm{a}$ & $23.27 \mathrm{~b}$ \\
\hline D5 & $40.00 \mathrm{a}$ & $11.53 \mathrm{a}$ & $12.70 \mathrm{a}$ & $23.05 \mathrm{~b}$ \\
\hline $\mathrm{LSD}_{0.05}$ & 4.37 & 0.56 & 13.02 & 0.98 \\
\hline CV. $(\%)$ & 9.51 & 1.22 & 28.36 & 19.60 \\
\hline \multicolumn{5}{|l|}{ Cutting date } \\
\hline $\mathrm{C}_{1}$ & $36.15 b$ & $11.15 b$ & $122.96 a$ & $23.20 \mathrm{a}$ \\
\hline $\mathrm{C}_{2}$ & $39.80 \mathrm{ab}$ & $11.55 \mathrm{ab}$ & $107.95 \mathrm{a}$ & $23.21 \mathrm{a}$ \\
\hline $\mathrm{C}_{3}$ & $45.90 \mathrm{a}$ & $11.65 \mathrm{ab}$ & $122.83 a$ & $24.43 \mathrm{a}$ \\
\hline $\mathrm{C}_{4}$ & $42.15 \mathrm{ab}$ & $12.28 \mathrm{a}$ & $114.74 \mathrm{a}$ & $23.54 \mathrm{a}$ \\
\hline $\mathrm{LSD}_{0.05}$ & 3.93 & 0.47 & 0.91 & 2.90 \\
\hline CV. $(\%)$ & 7.92 & 0.96 & 22.32 & 6.19 \\
\hline
\end{tabular}

a and b compared with LSD $(\mathrm{P}<0.05)$

${ }^{1 /} \mathrm{D}_{1}=$ control, (without leaf removal), $\mathrm{D}_{2}=$ defoliating all leaves, $\mathrm{D}_{3}=$ defoliating leaves under the ear, $\mathrm{D}_{4}=$ remain 2 top leaves, $\mathrm{D}_{5}=$ remain ear leaf

${ }^{1 /} \mathrm{C}_{1}=7$ days after silking, $\mathrm{C}_{2}=10$ days after silking, $\mathrm{C}_{3}=13$ days after silking and $\mathrm{C}_{4}=16$ days after silking

Table 2: The interaction of the defoliation patterns and leaves cutting date on seed number per row during 2018 (year 1)

\section{Seed number per row}

Cutting date

\begin{tabular}{lllll} 
Defoliation & $\mathrm{C}_{1}$ & $\mathrm{C}_{2}$ & $\mathrm{C}_{3}$ & $\mathrm{C}_{4}$ \\
\hline $\mathrm{D}_{1}$ & $14.98 \mathrm{ab}$ & $15.43 \mathrm{a}$ & $15.30 \mathrm{a}$ & $12.16 \mathrm{abcd}$ \\
$\mathrm{D}_{2}$ & $6.25 \mathrm{e}$ & $8.38 \mathrm{de}$ & $9.83 \mathrm{cde}$ & $14.61 \mathrm{ab}$ \\
$\mathrm{D}_{3}$ & $13.08 \mathrm{abc}$ & $16.38 \mathrm{a}$ & $14.00 \mathrm{abc}$ & $13.56 \mathrm{abc}$ \\
$\mathrm{D}_{4}$ & $13.56 \mathrm{abc}$ & $15.53 \mathrm{a}$ & $12.25 \mathrm{abcd}$ & $4.65 \mathrm{f}$ \\
$\mathrm{D}_{5}$ & $16.45 \mathrm{a}$ & $13.35 \mathrm{abc}$ & $10.50 \mathrm{bcde}$ & $10.50 \mathrm{bcde}$ \\
LSD 0.05 & 2.04 & & & \\
CV. $(\%)$ & 4.12 & & & \\
\hline
\end{tabular}

$\mathrm{a}, \mathrm{b}, \mathrm{c}, \mathrm{d}, \mathrm{e}$ and $\mathrm{f}$ compared with LSD $(\mathrm{P}<0.05)$

${ }^{1 /} \mathrm{D}_{1}=$ control, (without leaf removal), $\mathrm{D}_{2}=$ defoliating all leaves, $\mathrm{D}_{3}=$ defoliating leaves under the ear, $\mathrm{D}_{4}=$ remain 2 top leaves, $\mathrm{D}_{5}=$ remain ear leaf

${ }^{1 /} \mathrm{C}_{1}=7$ days after silking, $\mathrm{C}_{2}=10$ days after silking, $\mathrm{C}_{3}=13$ days after silking and $\mathrm{C}_{4}=16$ days after silking

Table 3: Effect of defoliation patterns and leaf cutting date on maize seed germination traits during 2018 (year 1)

\begin{tabular}{|c|c|c|c|c|c|c|}
\hline Treatment $^{1 /}$ & $\begin{array}{l}\text { Germination, } \\
\%\end{array}$ & $\begin{array}{l}\text { Shoot length, } \\
\mathrm{cm}\end{array}$ & $\begin{array}{l}\text { Root length, } \\
\mathrm{cm}\end{array}$ & $\begin{array}{l}\text { Seedling } \\
\text { growth rate }\end{array}$ & $\begin{array}{l}\text { Speed of } \\
\text { germination }\end{array}$ & $\begin{array}{l}\text { AA - test } \\
\text { germination at } 6^{\text {th }} \text { month }\end{array}$ \\
\hline \multicolumn{7}{|l|}{ Defoliation } \\
\hline$D_{1}$ & $90.78 \mathrm{a}$ & $10.42 \mathrm{ab}$ & $6.72 b$ & $2.15 \mathrm{ab}$ & $22.50 \mathrm{a}$ & $87.50 \mathrm{ab}$ \\
\hline $\mathrm{D}_{2}$ & $58.25 \mathrm{c}$ & $6.79 c$ & $8.60 \mathrm{a}$ & $1.98 \mathrm{c}$ & $17.51 \mathrm{c}$ & $59.75 d$ \\
\hline $\mathrm{D}_{3}$ & $92.25 \mathrm{a}$ & $11.22 \mathrm{a}$ & $6.72 b$ & $2.21 \mathrm{a}$ & $22.90 \mathrm{a}$ & $90.14 \mathrm{a}$ \\
\hline $\mathrm{D}_{4}$ & $80.53 b$ & $7.91 \mathrm{~cd}$ & 7.99ab & $2.06 \mathrm{bc}$ & $20.78 b$ & $80.38 b c$ \\
\hline $\mathrm{D}_{5}$ & $74.25 b$ & $8.75 b c$ & $7.69 \mathrm{ab}$ & $2.14 \mathrm{ab}$ & $20.91 b$ & $76.75 \mathrm{c}$ \\
\hline $\mathrm{LSD}_{0.05}$ & 5.56 & 0.88 & 0.69 & 0.06 & 0.63 & 5.86 \\
\hline CV. (\%) & 24.10 & 1.92 & 1.52 & 0.13 & 1.36 & 12.17 \\
\hline \multicolumn{7}{|l|}{ Cutting date } \\
\hline $\mathrm{C}_{1}$ & $81.00 \mathrm{a}$ & $8.25 a$ & $7.69 a$ & $2.03 \mathrm{a}$ & $20.89 a$ & $73.20 \mathrm{a}$ \\
\hline $\mathrm{C}_{2}$ & $86.00 \mathrm{a}$ & $9.07 \mathrm{a}$ & $7.93 \mathrm{a}$ & $2.21 \mathrm{a}$ & $20.63 a$ & $71.80 \mathrm{a}$ \\
\hline $\mathrm{C}_{3}$ & $64.00 \mathrm{~b}$ & $9.03 \mathrm{a}$ & $7.25 \mathrm{a}$ & $2.06 \mathrm{a}$ & $21.63 \mathrm{a}$ & $83.80 \mathrm{a}$ \\
\hline $\mathrm{C}_{4}$ & $78.20 \mathrm{ab}$ & $9.72 \mathrm{a}$ & $7.45 \mathrm{a}$ & $2.14 \mathrm{a}$ & $20.92 a$ & $66.40 \mathrm{a}$ \\
\hline $\mathrm{LSD}_{0.05}$ & 9.29 & 0.73 & 0.54 & 0.09 & 0.59 & 6.42 \\
\hline CV. $(\%)$ & 19.80 & 1.48 & 1.10 & 0.19 & 1.19 & 10.15 \\
\hline
\end{tabular}

$\mathrm{a}, \mathrm{b}, \mathrm{c}$ and d compared with LSD $(\mathrm{P}<0.05)$

${ }^{1 /} \mathrm{D}_{1}=$ control, (without leaf removal), $\mathrm{D}_{2}=$ defoliating all leaves, $\mathrm{D}_{3}=$ defoliating leaves under the ear, $\mathrm{D}_{4}=$ remain 2 top leaves, $\mathrm{D}_{5}=$ remain ear leaf

${ }^{1 /} \mathrm{C}_{1}=7$ days after silking, $\mathrm{C}_{2}=10$ days after silking, $\mathrm{C}_{3}=13$ days after silking and $\mathrm{C}_{4}=16$ days after silking 
Table 4: Effect of defoliation patterns and leaf cutting date on maize yield and yield components during 2019 (year 2)

\begin{tabular}{|c|c|c|c|c|}
\hline Treatment $^{1 /}$ & Ear weight, g/plant b & Row number per ear & Seed number per row & $100-$ Seed weight \\
\hline \multicolumn{5}{|l|}{ Defoliation } \\
\hline $\mathrm{D}_{1}$ & $62.01 \mathrm{a}$ & $11.61 \mathrm{ab}$ & $13.18 \mathrm{ab}$ & $26.04 \mathrm{a}$ \\
\hline $\mathrm{D}_{2}$ & $29.80 \mathrm{~b}$ & $9.98 \mathrm{~d}$ & $9.99 \mathrm{c}$ & $17.28 \mathrm{~d}$ \\
\hline $\mathrm{D}_{3}$ & $61.71 \mathrm{a}$ & $12.21 \mathrm{a}$ & $13.80 \mathrm{a}$ & $26.20 \mathrm{a}$ \\
\hline $\mathrm{D}_{4}$ & $30.80 \mathrm{~b}$ & $10.57 \mathrm{~cd}$ & $11.57 \mathrm{bc}$ & $23.62 b$ \\
\hline D5 & $38.75 b$ & $11.26 \mathrm{bc}$ & $12.78 \mathrm{ab}$ & $22.71 \mathrm{c}$ \\
\hline $\mathrm{LSD}_{0.05}$ & 7.15 & 0.38 & 0.79 & 0.40 \\
\hline CV. $(\%)$ & 15.58 & 0.84 & 1.74 & 0.88 \\
\hline \multicolumn{5}{|l|}{ Cutting date } \\
\hline $\mathrm{C}_{1}$ & $38.11 \mathrm{~b}$ & $11.10 \mathrm{a}$ & $12.54 \mathrm{ab}$ & $22.85 \mathrm{a}$ \\
\hline $\mathrm{C}_{2}$ & $43.50 \mathrm{ab}$ & $10.85 \mathrm{a}$ & $12.28 \mathrm{ab}$ & $23.18 \mathrm{a}$ \\
\hline $\mathrm{C}_{3}$ & $53.36 \mathrm{a}$ & $11.66 \mathrm{a}$ & $13.33 \mathrm{a}$ & $23.19 \mathrm{a}$ \\
\hline $\mathrm{C}_{4}$ & $43.49 \mathrm{ab}$ & $10.88 \mathrm{a}$ & $10.91 b$ & $23.46 \mathrm{a}$ \\
\hline $\mathrm{LSD}_{0.05}$ & 4.98 & 0.50 & 1.08 & 0.49 \\
\hline CV. $(\%)$ & 10.04 & 1.00 & 2.17 & 0.99 \\
\hline
\end{tabular}

a and b compared with LSD $(\mathrm{P}<0.05)$

${ }^{1 /} \mathrm{D}_{1}=$ control, (without leaf removal), $\mathrm{D}_{2}=$ defoliating all leaves, $\mathrm{D}_{3}=$ defoliating leaves under the ear, $\mathrm{D}_{4}=$ remain 2 top leaves, $\mathrm{D}_{5}=$ remain ear leaf.

${ }^{1 /} \mathrm{C}_{1}=7$ days after silking, $\mathrm{C}_{2}=10$ days after silking, $\mathrm{C}_{3}=13$ days after silking and $\mathrm{C}_{4}=16$ days after silking

Table 5: The interaction of the defoliation patterns and leaves cutting date on row number per ear during 2019 (year 2)

\begin{tabular}{|c|c|c|c|c|}
\hline \multirow[b]{2}{*}{ Defoliation } & \multicolumn{4}{|c|}{$\begin{array}{l}\text { Row number per ear } \\
\text { Cutting date }\end{array}$} \\
\hline & $\mathrm{C}_{1}$ & $\mathrm{C}_{2}$ & $\mathrm{C}_{3}$ & $\mathrm{C}_{4}$ \\
\hline$\overline{D_{1}}$ & $12.06 \mathrm{abc}$ & $11.05 \mathrm{abcd}$ & $12.02 \mathrm{abc}$ & $11.30 \mathrm{abcd}$ \\
\hline $\mathrm{D}_{2}$ & $10.33 \mathrm{~cd}$ & $10.03 \mathrm{cde}$ & $9.24 \mathrm{de}$ & $10.33 \mathrm{~cd}$ \\
\hline $\mathrm{D}_{3}$ & $11.73 \mathrm{abc}$ & $12.65 \mathrm{ab}$ & $13.00 \mathrm{a}$ & $11.45 \mathrm{abc}$ \\
\hline $\mathrm{D}_{4}$ & $10.78 \mathrm{bcd}$ & $7.95 \mathrm{e}$ & $12.46 \mathrm{ab}$ & $11.09 \mathrm{abcd}$ \\
\hline $\mathrm{D}_{5}$ & $10.60 \mathrm{bcd}$ & $12.558 \mathrm{ab}$ & $11.60 \mathrm{abc}$ & $10.25 \mathrm{~cd}$ \\
\hline LSD 0.05 & 1.12 & & & \\
\hline CV. (\%) & 2.26 & & & \\
\hline
\end{tabular}

a,b,c,d and e compared with LSD $(\mathrm{P}<0.05)$

${ }^{1 /} \mathrm{D}_{1}=$ control, (without leaf removal), $\mathrm{D}_{2}=$ defoliating all leaves, $\mathrm{D}_{3}=$ defoliating leaves under the ear, $\mathrm{D}_{4}=$ remain 2 top leaves, $\mathrm{D}_{5}=$ remain ear leaf

${ }^{1 /} \mathrm{C}_{1}=7$ days after silking, $\mathrm{C}_{2}=10$ days after silking, $\mathrm{C}_{3}=13$ days after silking and $\mathrm{C}_{4}=16$ days after silking

Table 6: Effect of defoliation patterns and leaf cutting date on maize seed germination traits during 2019 (year 2)

\begin{tabular}{|c|c|c|c|c|c|c|}
\hline Treatment $^{1 /}$ & $\begin{array}{l}\text { Germination, } \\
\%\end{array}$ & $\begin{array}{l}\text { Shoot length, } \\
\mathrm{cm}\end{array}$ & $\begin{array}{l}\text { Root length, } \\
\mathrm{cm}\end{array}$ & $\begin{array}{l}\text { Seedling } \\
\text { growth rate }\end{array}$ & $\begin{array}{l}\text { Speed of } \\
\text { germination }\end{array}$ & $\begin{array}{l}\text { AA - test } \\
\text { germination at } 6^{\text {th }} \text { month }\end{array}$ \\
\hline \multicolumn{7}{|l|}{ Defoliation } \\
\hline $\mathrm{D}_{1}$ & $89.19 \mathrm{a}$ & $9.65 \mathrm{ab}$ & $6.75 b c$ & $1.47 \mathrm{a}$ & $21.90 \mathrm{a}$ & $83.19 \mathrm{a}$ \\
\hline $\mathrm{D}_{2}$ & $70.31 \mathrm{c}$ & $6.22 \mathrm{c}$ & $8.58 \mathrm{a}$ & $1.23 \mathrm{c}$ & $17.75 \mathrm{c}$ & $64.31 \mathrm{c}$ \\
\hline $\mathrm{D}_{3}$ & $91.31 \mathrm{a}$ & $10.41 \mathrm{a}$ & $6.52 \mathrm{c}$ & $1.50 \mathrm{a}$ & $22.59 \mathrm{a}$ & $85.31 \mathrm{a}$ \\
\hline $\mathrm{D}_{4}$ & $70.88 \mathrm{c}$ & $7.23 c$ & 7.86abc & $1.32 \mathrm{bc}$ & $17.42 \mathrm{c}$ & $64.88 \mathrm{c}$ \\
\hline $\mathrm{D}_{5}$ & $76.69 \mathrm{~b}$ & $7.94 b c$ & 7.98ab & $1.41 \mathrm{ab}$ & $18.90 \mathrm{~b}$ & $70.69 b$ \\
\hline $\mathrm{LSD}_{0.05}$ & 1.19 & 0.94 & 0.65 & 0.05 & 0.50 & 1.16 \\
\hline CV. $(\%)$ & 2.60 & 2.05 & 1.41 & 0.12 & 1.10 & 2.60 \\
\hline \multicolumn{7}{|l|}{ Cutting date } \\
\hline $\mathrm{C}_{1}$ & $78.35 \mathrm{c}$ & $7.53 b$ & 7.70a & $1.29 b$ & $19.24 b$ & $72.35 \mathrm{c}$ \\
\hline $\mathrm{C}_{2}$ & $79.45 b c$ & $8.44 \mathrm{ab}$ & 7.78a & $1.51 \mathrm{a}$ & $19.61 b$ & $73.45 b c$ \\
\hline $\mathrm{C}_{3}$ & $80.95 \mathrm{a}$ & $8.09 \mathrm{ab}$ & $7.36 \mathrm{a}$ & $1.33 \mathrm{ab}$ & $20.36 \mathrm{a}$ & $74.95 \mathrm{a}$ \\
\hline $\mathrm{C}_{4}$ & 79.95ab & $9.10 \mathrm{a}$ & $7.30 \mathrm{a}$ & $1.42 \mathrm{ab}$ & $19.65 b$ & 73.95ab \\
\hline $\mathrm{LSD}_{0.05}$ & 0.72 & 0.78 & 0.53 & 0.09 & 0.32 & 0.51 \\
\hline CV. $(\%)$ & 1.45 & 1.54 & 1.08 & 0.19 & 0.65 & 1.05 \\
\hline
\end{tabular}

a, b, c and d compared with LSD $(\mathrm{P}<0.05)$

${ }^{1 /} \mathrm{D}_{1}=$ control, (without leaf removal), $\mathrm{D}_{2}=$ defoliating all leaves, $\mathrm{D}_{3}=$ defoliating leaves under the ear, $\mathrm{D}_{4}=$ remain 2 top leaves, $\mathrm{D}_{5}=$ remain ear leaf

${ }^{1 /} \mathrm{C}_{1}=7$ days after silking, $\mathrm{C}_{2}=10$ days after silking, $\mathrm{C}_{3}=13$ days after silking and $\mathrm{C}_{4}=16$ days after silking 


\section{Shoot Length and Root Length}

Defoliation treatments had significantly impact on seedling shoot length and root length in both years 2018 and 2019 (Table 3 and 6). There had a negative correlation between root length reduced while shoot length, seedling growth rate and germination percent increased (Heidari, 2012).

\section{Seed Vigor}

The significantly effect of defoliation treatments on seedling weight and vigor was shown in both years 2018 and 2019 (Table 3 and 6). $\mathrm{D}_{3}$ the best increased high seedling growth rate, speed of germination and AA test germination percentage after $6^{\text {th }}$ month of storage it's not significantly different from $\mathrm{D}_{1}$. While the $\mathrm{D}_{2}, \mathrm{D}_{4}$ and $\mathrm{D}_{5}$ still show low seedling growth rate, speed of germination and AA test germination percentage after at $6^{\text {th }}$ month of storage when compared with control which was no leaf removal (Table 3 and 6).

Defoliation leaves under ear severely $\left(\mathrm{D}_{3}\right)$ increased 100 - seeds weight, seed germination percentage, speed of germination and seed vigor. This might be due to those defoliating leaves under ear acting as a parasitic or metabolic sink that competed for ear and kernel development during the grain filling period. Those leaves at below part of the maize stem and upper leaves could provided shade for the leaves in the central position (Heidari, 2012). A senescent leaf undoubtedly reduces the supply of photosynthate available for distribution to the grain developing as indicated by the decline in stem weight and carbohydrate concentration (Jones and Simmons, 1983). If the defoliation leaves under ear severely, the quantity of retransferred assimilation from stem to grain would be increased. Defoliating leaves below the ear did not significantly decrease corn yield and seed quality (Koptur, et al., 1996).

Complete defoliation $\left(\mathrm{D}_{2}\right)$ caused the decreasing in yield and yield components. As same as study on grain maize showed, complete defoliation caused to diminish of the yield about 95\% (Melchiori and Caviglia, 2008). Defoliation leaves on top of the ear $\left(\mathrm{D}_{4}\right.$ and $\left.\mathrm{D}_{5}\right)$ caused more impact which decreased in the rate of grain filling because of only remained leaves were unavailable to supply enough to requirement of assimilate for plant. The effective period of grain filling had greater effected increasingly by defoliation than the rate of grain filling. The results suggest that the top leaves should be prevent for defoliate, because this treatment showed the negative effect on yield (Heidari, 2012). As reported by (Borrás et al., 2004), a decreasing of the post-flowering source/sink ratio could reduce final kernel weight dramatically. Matthews, (1973) reported large seed size could promoted the higher germination percentage ( $48 \%$ ) while small seed size gave a lower germination percentage $(46.0 \%)$ as same as the observing that germination was higher $(89.6 \%)$ in large sized seeds and lower (85.2\%) in small sized seeds.
Storability of seeds was a major genetic characteristic and was influenced by pre-storage history of seeds, seed maturation and environmental factors during both of preharvest and post-harvest (Tuite and Foster, 1979).

\section{Conclusion and Suggestions}

The results of this study showed defoliating leaves under ear $\left(D_{3}\right)$ could maintain seed storability with high seed germination percentage and seed vigor. The result in maize seeds had the potential to grow to normal seedlings in the field condition, compared to removing the top of the ear $\left(D_{4}\right.$ and $\left.D_{5}\right)$. Therefore, improving the seed quality in maize, hybrids and agronomic practices should focus to promote the post-flowering source/sink ratio. The recommendation is to study the effect of other environmental factors such as light by removing leaves under and at the top of ear on seed qualities and storability is remarkably interesting. Finally, leaf defoliation at upper of ear was more impact to all investigated characteristics as well as the results that suggested the upper leaves should not defoliate, because this treatment has negative effect on the yield.

\section{Acknowledgment}

This work was supported by Syngenta Seeds (Thailand) Co. Ltd. for the source of maize seeds. We are grateful to farmer field at U-Thong district, Suphan Buri province for place of planting. Thank you Department of Agronomy, Faculty of Agriculture, Kasetsart University for working place at Crop Physiology and Renewable Energy Crops Laboratory, Seed laboratory and Prof. Dr. Michael Bredemeier at Georg - August University of Gottingen, Germany for supporting.

\section{References}

Borrás, L., \& Otegui, M. E. (2001). Maize kernel weight response to postflowering source-sink ratio. Crop Science, 41(6), 1816-1822. https://doi.org/10.2135/cropsci2001.1816

Borrás, L., Curá, J. A., \& Otegui, M. E. (2002). Maize kernel composition and post-flowering source-sink ratio. Crop Science, 42(3), 781-790. https://doi.org/10.2135/cropsci2002.0781

Borrás, L., Slafer, G. A., \& Otegui, M. E. (2004). Seed dry weight response to source-sink manipulations in wheat, maize and soybean: a quantitative reappraisal. Field Crops Research, 86(2-3), 131-146. https://doi.org/10.1016/j.fcr.2003.08.002

Borrás, L., Zinselmeier, C., Senior, M. L., Westgate, M. E., \& Muszynski, M. G. (2009). Characterization of grain-filling patterns in diverse maize germplasm. Crop Science, 49(3), 999-1009. https://doi.org/10.2135/cropsci2008.08.0475 
Collantes, H. G., Gianoli, E., \& Niemeyer, H. M. (1997). Effect of defoliation on the patterns of allocation of a hydroxamic acid in rye (Secale cereale). Environmental and Experimental Botany, 38(3), 231-235. https://doi.org/10.1016/S0098-8472(97)00009-9

Gambín, B. L., Borrás, L., \& Otegui, M. E. (2006). Source-sink relations and kernel weight differences in maize temperate hybrids. Field Crops Research, 95(2-3), 316-326. https://doi.org/10.1016/j.fcr.2005.04.002

Gifford, R. M, Thorne, J. H., Hitz, W. D., \& Giaquinta, R. T. (1984). Crop productivity and photoassimilate partitioning. Science, 225, 801-808. https://science.sciencemag.org/content/225/4664/801

Heidari, H. (2012). Effect of defoliation intensity on maize yield, yield components and seed germination. Life Science Journal, 9 (4), 1587-1590. http://citeseerx.ist.psu.edu/viewdoc/download?doi=1 0.1.1.448.6751\&rep=rep1\&type $=$ pdf

ISTA. (2020). International rules for seed testing 2021. International Seed Testing Association. Zurich, Switzerland.

https://www.seedtest.org/en/international-rules-forseed-testing-_content---1--1083.html

Jones, R. J., \& Simmons, S. R. (1983). Effect of altered source-sink ratio on growth of maize kernels. Crop Science, 23, 129-134. https://acsess.onlinelibrary.wiley.com/doi/abs/10.21 35/cropsci1983.0011183X002300010038x

Khatun, A., Kabir, G., \& Bhuiyan, M. A. H. (2009). Effect of harvesting stages on the seed quality of lentil (Lens culinaris L.) during storage. Bangladesh Journal of Agricultural Research, 34(4), 565-576. https://doi.org/10.3329/bjar.v34i4.5833

Koptur, S., Smith, C. L., \& Lawton, J. H. (1996). Effects of artificial defoliation on reproductive allocation in the common vetch Vicia sativa (fabaceae; papilionoideae). American Journal of Botany, 83(7), 886-889. https://doi.org/10.2307/2446265
Matthews, S. (1973). The effect of time of harvest on the viability and pre-emergence mortality in soil of pea (Pisutn sativum L.) seeds. Annals of Applied Biology, 73(2), 211-219. https://doi.org/10.1111/j.1744-7348.1973.tb01327.x

Mehta, C. J., Kuhad, M. S., Sheoran, I. S., \& Nandwal, A. S. (1993). Studies on seed development and germination in chickpea cultivars. Seed Research, 21(2), 89-91.

Melchiori, R. J. M., \& Caviglia, O. P. (2008). Maize kernel growth and kernel water relations as affected by nitrogen supply. Field Crops Research, 108, 198-205. https://doi.org/10.1016/j.fcr.2008.05.003

Shaheb, M. R., Islam, M. N., Nessa, A., \& Hossain, M. A. (2015). Effect of harvest times on the yield and seed quality of French bean. Saarc Journal of Agriculture, 13(1), 1-13. https://doi.org/10.3329/sja.v13i1.24175

Tuite, J., \& Foster, G. H. (1979). Control of storage diseases of grain. Annual Review of Phytopathology, $17(1)$ 343-366. https://doi.org/10.1146/annurev.py.17.090179.002015

Uhart, S. A., \& Andrade, F. H. (1991). Source-sink relationships in maize grown in a cool-temperate area. Agronomie, 11(10), 863-875. https://doi.org/10.1051/agro:19911004

Uhart, S. A., \& Andrade, F. H. (1995). Nitrogen and carbon accumulation and remobilization during grain filling in maize under different source/sink ratios. Crop Science, 35, 183-190. https://acsess.onlinelibrary.wiley.com/doi/abs/10.21 35/cropsci1995.0011183X003500010034x

van den Boogaard, R., Grevsen, K., \& Thorup-Kristensen, K. (2001). Effects of defoliation on growth of cauliflower. Scientia Horticulturae, 91(1-2), 1-16. https://doi.org/10.1016/S0304-4238(01)00234-5 